\title{
Time-intensity Curve Analysis of Hepatocellular Carcinoma using Two Contrast-enhanced Ultrasound Methods: Contrast Pulse Sequencing and Contrast Harmonic Imaging
}

\author{
Mengna He, MD, PhD ${ }^{a}$, Lei Xu, MD ${ }^{b}$, Tian'an Jiang, $M D, P h D^{a, *}$ \\ ${ }^{a}$ Department of Ultrasound, The First Affiliated Hospital of Zhejiang University, Hangzhou, Zhejiang, China; ${ }^{b}$ Department of \\ Ultrasound, Jinhua Hospital of Zhejiang University, Jinhua, Zhejiang, China
}

Received February 12, 2020; revision received May 30, 2020; accepted May 31, 2020.

\begin{abstract}
Objectives: To compare the features of the time-intensity curve (TIC) of hepatocellular carcinoma (HCC) by two different contrast-enhanced ultrasound (CEUS) methods: Contrast Pulse Sequencing (CPS) and Contrast Harmonic Imaging (CHI).

Methods: This prospective study included 22 HCC lesions. The CPS and CHI (Cadence ${ }^{\mathrm{TM}}$ technique by Siemens) were performed in random order for each lesion, and the microbubbles were completely cleared between the two methods. The imaging by each method was recorded for 10 minutes. The CEUS video clips were analyzed off-line and the quantitative parameters of time intensity curve were obtained: the peak intensity (PI), time to peak (TTP), washout time (WT), relative value (RV) of intensity and AUC before WT and after WT, and the time of RV $\geqslant 15 \mathrm{~dB}$ lasted (RLT).
\end{abstract}

Results: Compared with CPS, CHI showed an earlier WT ( $64.0 \pm 17.1 \mathrm{~s}$ vs $33.1 \pm 7.0 \mathrm{~s})$ of HCC lesions, a lower RV of intensity $(36.8 \pm 9.4$ vs $10.3 \pm 5.1)$ and AUC $(1377.2 \pm 205.7$ vs $227.2 \pm 56.7)$ before WT, but higher RV of intensity $(17.8 \pm 4.6$ vs $32.2 \pm$ 8.6) and AUC ( $1124.1 \pm 276.4$ vs $2664.1 \pm 456.8)$ after WT, and longer RLT $(121.4 \pm 49.8 \mathrm{~s}$ vs > $150 \mathrm{~s})$.

Conclusion: For long later phase observation after washout, $\mathrm{CHI}$ is better than CPS, but the observation of rapid perfusion before washout is not comparable to CPS. A combined use of these two methods is recommended based on our research.

Key words: Contrast enhanced ultrasound; Contrast pulse sequence, Contrast harmonic imaging; Time-intensity curve; Hepatocellular carcinoma

Advanced Ultrasound in Diagnosis and Therapy 2020;03:217-222

DOI: 10.37015/AUDT.2020.200007

$\mathrm{H}$ epatocellular carcinoma (HCC) is the fourth leading cause of cancer-related deaths globally [1]. A worldwide epidemiologic research in 2012 showed China alone accounting for $50 \%$ of all newly diagnosed HCC, the morbidity of HCC in America and Europe was lower but present an increasing trend [2]. $70 \%$ of HCC cases were at an advanced stage at first diagnosis and their survival rate remained low, therefore, early detection of $\mathrm{HCC}$ is extremely important.
When HCC is primarily suspected, diagnostic imaging such as contrast-enhanced computer tomography (CE$\mathrm{CT}$ ) and contrast-enhanced magnetic resonance imaging (CE-MRI) are the first-line imaging examinations recommended for the cancer stage evaluation $[3,4]$. However, both of them require contrast media, which is not suitable for patients with extremely impaired renal function [5], besides, the radiation injury of CT cannot be ignored and MRI examination relatively takes a long time.

\footnotetext{
" Corresponding author: Department of Ultrasound, The First Affiliated Hospital of Zhejiang University; Qingchun Road, No.79, Hangzhou 310003, Zhejiang, China

e-mail: tiananjiang@zju.edu.cn unrestricted use, distribution and reproduction in any medium provided that the original work is properly attributed.
} 
The use of contrast-enhanced ultrasound (CEUS) is definitely recommended for HCC diagnosis, as it is implemented in the European CEUS-guidelines, national and international HCC guidelines as well as applicable for MILAN criteria [6]. However, CEUS has some inherited limitations such as short observation time, only one target lesion can be observed with one injection, single plane observation, beyond that, the biggest challenge of CEUS is unable to complete a whole-liver scan during the contrast imaging period.

The new CEUS method of Contrast Harmonic Imaging (CHI) uses the sophisticated 2-pulse phase inversion technique (Cadence ${ }^{\mathrm{TM}}$ by Siemens Healthineers, USA) to isolate the second harmonic signal to obtain the high spatial resolution and frame rate along with the excellent bubble longevity, so it may increase the CEUS observation time allowing to detect more lesions including hypo enhanced ones in the late phase. The conventional CEUS method of Cadence ${ }^{\mathrm{TM}}$ Contrast Pulse Sequencing (CPS) (Siemens) uses the powerful 3-pulse sequence to maximize the harmonic signals for the maximal tissue cancellation and depth advantage. Thus, how to effectively use CHI and CPS for each application is still need to explore. This prospective study is the first study to report a comparison of the timeintensity curve characteristics obtained using the CPS and $\mathrm{CHI}$ techniques for $\mathrm{HCC}$.

\section{Patients and Materials}

\section{Clinical patient recruitment}

Thirty patients with suspected HCC by conventional ultrasound from February 2019 to May 2019 in the First Affiliated Hospital of Zhejiang University were recruited for this study. The study protocol was explained to each patient and informed consent was signed by all participants. Inclusion criteria included a single lesion less than $5 \mathrm{~cm}$ and a maximum of 3 lesions with each was less than $3 \mathrm{~cm}$. Exclusion criteria included local tumor treatment of the lesion, patients younger than 18 years old and without pathological confirmation. If there were multiple lesions, the largest one was selected for the observation. Both of the CEUS methods were performed in random order for all of the target lesions. This study was approved by our Institute Ethics Review Committee (NO.2019016).

\section{Contrast enhanced ultrasound techniques}

The ACUSON Oxana 3 ultrasound imaging system (Siemens Healthineers, Mountain View, CA, USA) equipped with a 6C1 HD convex array transducer (1-6 $\mathrm{MHz}$ ) was used for examination, which allowed working with CPS and CHI mode. Some special parameters of
CPS and CHI modes were set respectively as follows, the Mechanical Index (MI) was 0.04 and 0.09, the frequency was $1.5 \mathrm{MHz}$ and $3.0 \mathrm{MHz}$. All of the ultrasound scanning were performed by the same radiologist with more than 10 years' experience in hepatic ultrasound.

All suspected lesions were evaluated by the grayscale ultrasound first, the patients were positioned supine and supplemented by the left decubitus after fasting for at least 6 hours, and the target lesion was identified and measured. Then, the two CEUS methods of CPS and CHI were performed in random order, about $2.4 \mathrm{ml}$ ultrasound contrast agent (SonoVue, Bracco, Italy) was injected as a bolus, followed by $5 \mathrm{ml}$ saline solution flush using a 20-gauge intravenous cannula. For each examination, at least 10 minutes of continuous realtime dynamic image were stored after the injection of SonoVue. Between the two examinations, we used Color Doppler Flow Imaging (CDFI) to burst the microbubbles and the interval time was at least 30 minutes until no obvious contrast agent was presented in the liver. During the examinations, patients were asked to breathe slowly and gently avoiding swallowing.

\section{Imaging analysis}

Sonoliver software (TomTec, Germany) was used for the off-line time-intensity curve (TIC) analysis on the dynamic images. The region of interest (ROI) was selected at least $20 \%$ of the whole mass, avoiding the necrotic area and large vessel, and the ROI of the normal liver parenchyma was selected at the same depth. Two radiologists with more than 10 years' experience in liver ultrasound who blinded to patients' clinical outcomes independently analyzed the dynamic images. All the necessary parameters were measured three times by each radiologist and the average value was finally calculated.

The following parameters were obtained from the TIC for this study: peak intensity (PI), time to peak (TTP), the elapsed time from the contrast agents entering the HCC lesion to reaching PI, washout time (WT), relative value $(\mathrm{RV})$ of intensity $(\Delta \mathrm{I})$, the max $\Delta \mathrm{I}$ before and after $\mathrm{WT}$, relative value (RV) of area under the time-intensity curve $(\triangle \mathrm{AUC})$, the $\triangle \mathrm{AUC}$ before $\mathrm{WT}$ and after $\mathrm{WT}$, and the time of relative value $(\mathrm{RV})$ of intensity $\geqslant 15 \mathrm{~dB}$ (RLT) of HCC and normal liver parenchyma lasted. The calculating method for $\triangle \mathrm{AUC}$ was described as Figure 1.

\section{Statistical analysis}

The statistical analysis was performed using SPSS17.0 (Chicago, USA), all continuous variables were described as mean \pm standard deviation. A paired t-test was applied for the comparison of TIC parameters from CPS and CHI. A value of $P<0.05$ was considered statistically significant. 


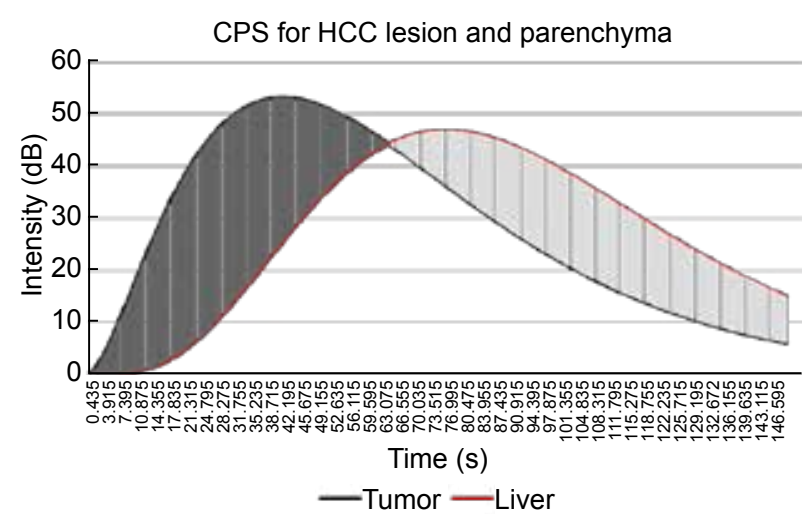

Figure 1 The calculating method for $\triangle \mathrm{AUC}$ was described, $\triangle \mathrm{AUC}$ should be calculated by integral with the formula as the following:

$$
\int_{0}^{\text {intercept }} f_{\text {tumor }}(t) d t-\int_{0}^{\text {intercept }} f_{\text {liver }}(t) d t
$$

As we don't have an explicit formula ftumor, fliver, the above integral can be calculated by numeric integration as the following:

$$
\sum_{i=1}^{n}\left(f_{\text {tumor }}\left(t_{i}\right)-f_{\text {tumor }}\left(t_{i-1}\right)\right) \times \frac{\Delta t}{2}-\sum_{i=1}^{n}\left(f_{\text {liver }}\left(t_{\mathrm{i}}\right)-f_{\text {liver }}\left(t_{i-1}\right)\right) \times \frac{\Delta t}{2}
$$

Where $i$ is the index of time axis, and $\Delta t$ is the time interval, which equals to $0.435 \mathrm{~s}$ in this case.

\section{Results}

Eventually 22 patients ( 4 females and 18 males) confirmed as HCC by pathology were included in our study for time-intensity curve analysis. The age of the patients ranged from 34 to 67 years $(58.4 \pm 10.2$ years). The size of the tumor ranged from 1.4 to $4.6 \mathrm{~cm}(2.0 \pm$ $0.8 \mathrm{~cm}$ ). Among the 22 patients, there were 7 patients with normal liver or mild to moderate fibrosis ( $2 \mathrm{~F} 0$, $2 \mathrm{~F} 1$, and $3 \mathrm{~F} 2$ ) and 15 patients with severe fibrosis (9 F3 and 6 F4). Three patients had well-differentiated HCC and 19 patients had low differentiated HCC. There were two misdiagnosed patients before biopsy, one was hepatic hemangioma and the other was inflammatory lesion suspected to be caused by parasitic infection (Fig. 2). Another 6 patients were excluded because they didn't meet the inclusion criteria.

The absolute and relative values from TIC were summarized in table 1 and table 2 respectively. Compared with CPS, CHI showed an earlier WT of HCC lesions, a lower max $\Delta \mathrm{I}$ and $\triangle \mathrm{AUC}$ before WT, but a
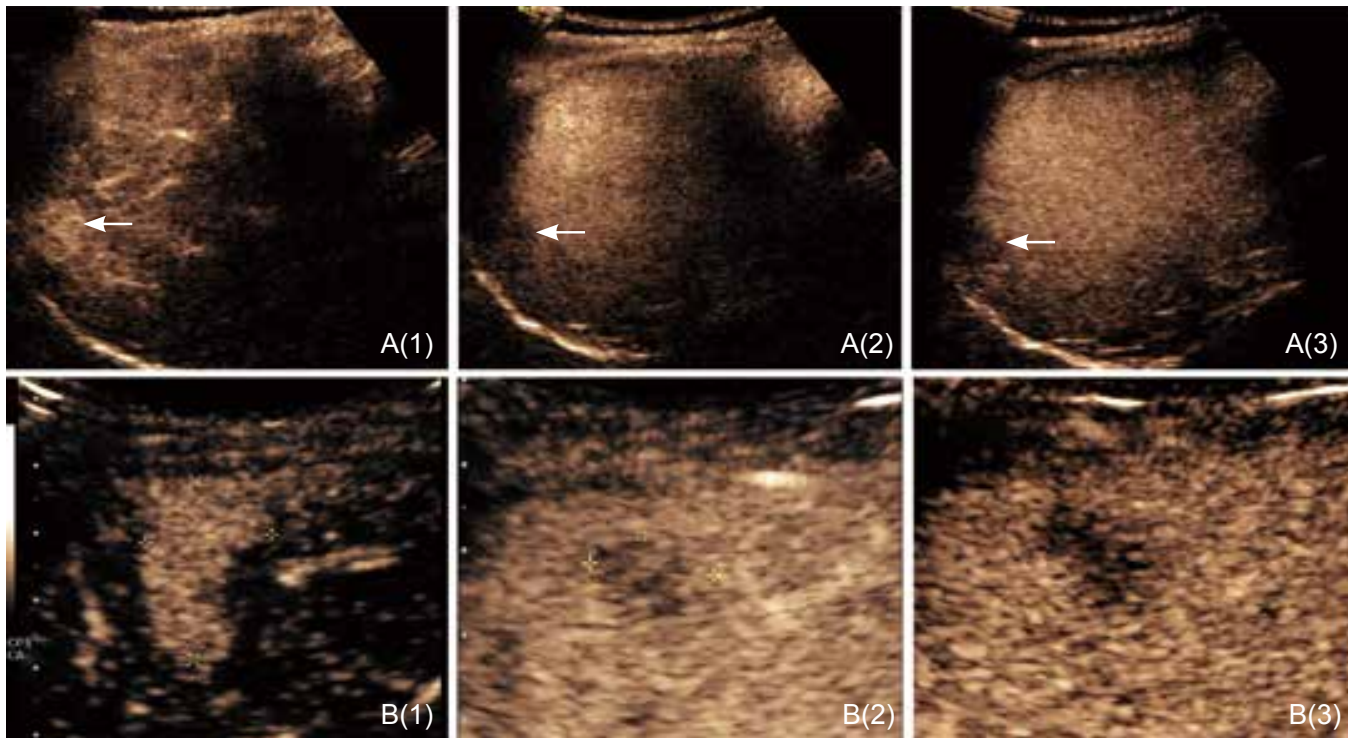

Figure 2 The CEUS manifestation of the two misdiagnosed cases. A (1-3) showed the CHI manifestation of a hepatic hemangioma of a 66-year-old women, the lesion was located under the capsule of the right posterior lobe (white arrow) with fast-in and fast-out enhancement pattern. B (1-3) showed the CPS manifestation of a parasitic inflammatory lesion of a 28 -year-old men, the lesion was located near the capsule of the right anterior lobe with fast-in and fast-out enhancement pattern.

higher max $\Delta \mathrm{I}$ and $\triangle \mathrm{AUC}$ after $\mathrm{WT}$, and a longer RLT $(P<0.05)$. The PI of the background liver parenchyma and HCC lesions from these two CEUS methods showed no statistically significant difference $(P>0.05)$. A typical HCC case with the manifestation of two CEUS methods were shown in Figure 3.

\section{Discussion}

Although both CPS and CHI were designed to solve the challenges in CEUS, but their priorities were different. Cadence CPS technology uses proprietary pulse sequencing technology that recognizes and processes the unique non-linear fundamental and high order harmonic signals generated by ultrasound contrast agents. While Cadence CHI isolated the 2nd harmonic signal to give the high resolution image. Both CPS and CHI were low MI technique, CPS was designed for performance at depth and CHI was for high spatial and temporal resolution. Besides, CPS has high sensitivity to contrast agent due to high signal-noise ratio, which was proven 

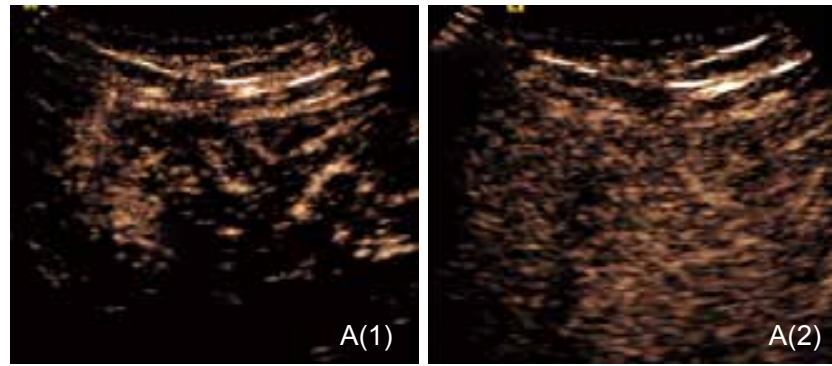

CPS for HCC lesion and parenchyma
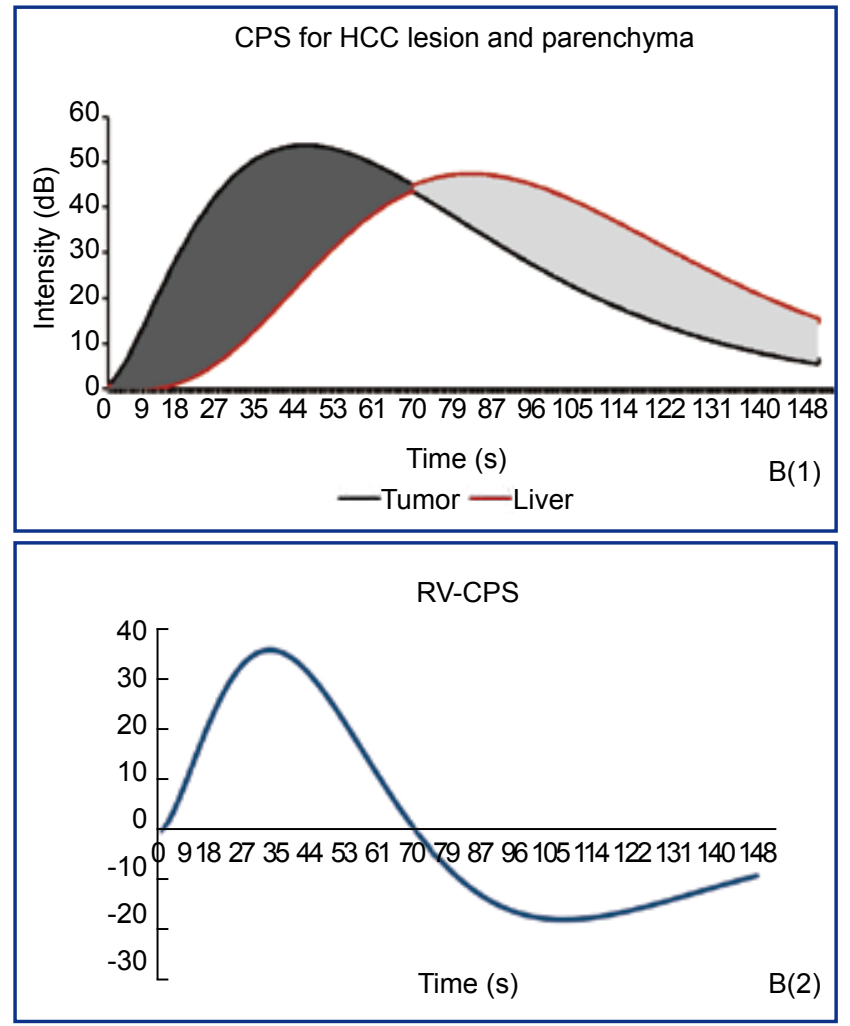
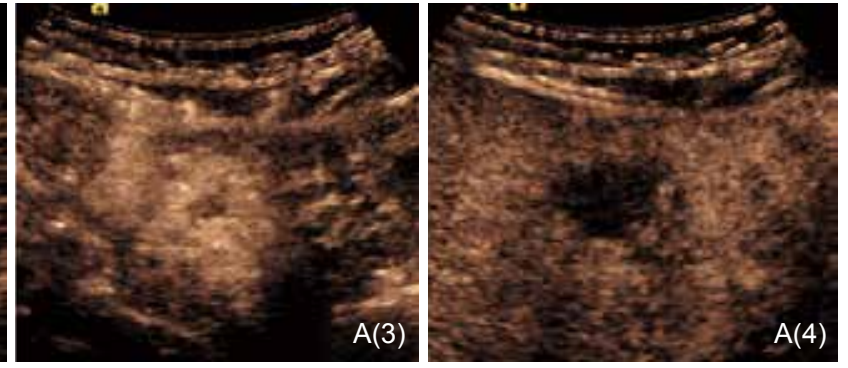

$\mathrm{CHI}$ for HCC lesion and parenchyma

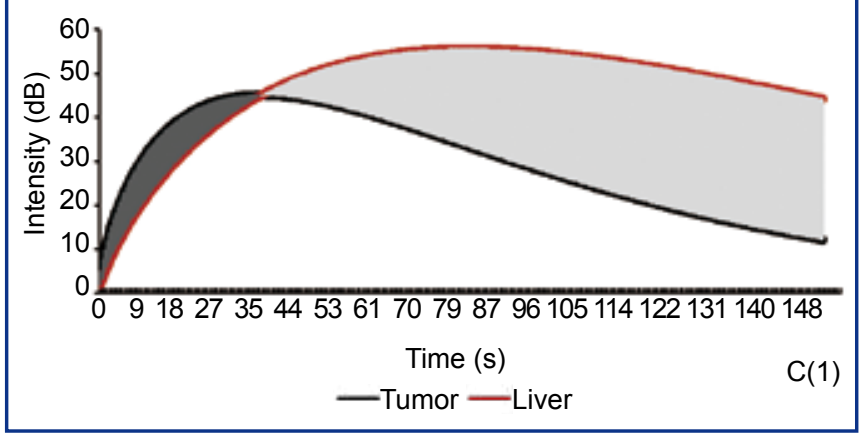

$\mathrm{RV}-\mathrm{CHI}$

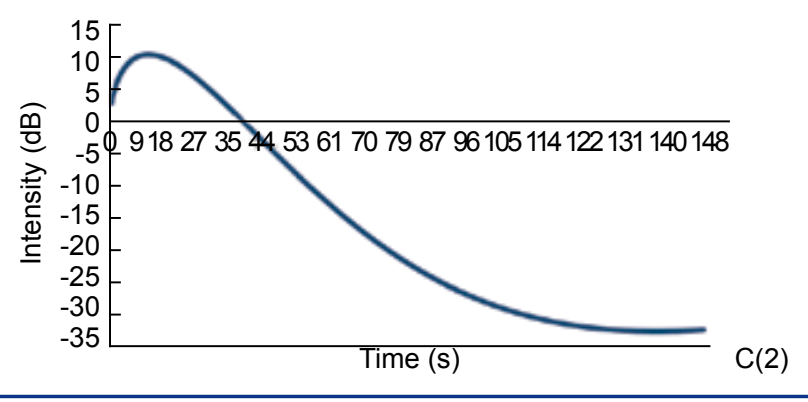

Figure 3 A (1-2) and A (3-4) showed the CPS and CHI manifestation of a pathology confirmed HCC lesion of a 65-year-old man, (1) arterial phase of $13 \mathrm{~s}$ after injection, the lesion was fast-in hyperenhanced, (2) later phase of $120 \mathrm{~s}$, the lesion presented hypo-enhancement, slightly lower than the liver parenchyma, (3) arterial phase of 16s after injection, the lesion was fast-in hyperenhanced, (4) later phase of 150s, the lesion presented low enhancement with clear margin with the liver parenchyma. B (1-2) and C (1-2) showed the TIC of the absolute intensity of HCC lesion and the liver parenchyma and the relative value of the intensity of CPS (B) and CHI (C).

Table 1 The comparison of absolute values from TIC of CHI and CPS.

\begin{tabular}{|c|c|c|c|c|c|c|}
\hline \multirow{2}{*}{ Item } & \multicolumn{3}{|c|}{ Hepatocellular carcinoma } & \multicolumn{3}{|c|}{ Liver } \\
\hline & $\mathrm{CHI}$ & CPS & $P$ value & $\mathrm{CHI}$ & CPS & $P$ value \\
\hline Peak intensity & $47.9 \pm 11.9$ & $53.1 \pm 14.0$ & 0.540 & $49.5 \pm 12.5$ & $46.8 \pm 13.2$ & 0.850 \\
\hline Time to peak & $33.5 \pm 13.5$ & $41.3 \pm 9.0$ & 0.005 & $65.8 \pm 13.2$ & $61.4 \pm 10.8$ & 0.531 \\
\hline
\end{tabular}

TIC, time-intensity curve; $\mathrm{CHI}$, contrast harmonic imaging; CPS, contrast pulse sequence

by our study. One of the results showed that compared with CPS, CHI had an earlier TTP and WT, lower $\triangle \mathrm{AUC}$ and max $\triangle \mathrm{I}$ before WT. These differences can be explained that CPS uses non-linear imaging techniques that isolate specific harmonics from bubbles' signal from the contrast-enhanced unpaired artery, while CHI was designed to show all the details of contrast-enhanced feature including the low flow and small vasculature, which might sacrifice the vessel contrast difference between the lesion and normal liver parenchymal before WT. However, after WT, the signal of vessel contrast enhancement that can be extracted from the lesion quickly reduced, while the advantages of low-flow signals extracted by $\mathrm{CHI}$ appeared, as our results showed 
that after WT, CHI had a higher $\triangle \mathrm{AUC}$ and $\max \Delta \mathrm{I}$.

Besides, CHI allows excellent bubble longevity and provides detailed imaging. According to our study, the time of relative value (RV) of intensity $\geqslant 15 \mathrm{~dB}$ between $\mathrm{HCC}$ and normal liver parenchyma lasted $>150 \mathrm{~s}$ under $\mathrm{CHI}$ while it lasted about $121.4 \mathrm{~s}$ on average under CPS during the 10-minute contrast period. Besides, $\triangle \mathrm{AUC}$ and max $\Delta \mathrm{I}$ after WT were much greater than CPS, implying that the intensity differences between HCC lesion and normal liver parenchyma of CHI was more significant than those of CPS in the phase after WT. These priorities will provide high contrast effect and enough time for the whole liver scan, some small and hidden HCC lesions may have a chance to be detected, long effective visual observation time after WT of CEUS also guaranteed adequate time for preoperative needle placement before the ablation of HCC.

Table 2 The comparison between the relative values from TIC of CHI and CPS

\begin{tabular}{lccc}
\hline & & Comprehensive parameters & \\
\cline { 2 - 4 } & Contrast Harmonic Imaging & Contrast Pulse Sequence & $P$ value \\
\hline WT(s) & $33.1 \pm 7.0$ & $64.0 \pm 17.1$ & 0.003 \\
\hline RLT(s) & $>150$ & $121.4 \pm 49.8$ & $<0.001$ \\
Before M $\Delta \mathrm{I}(\mathrm{dB})$ & $10.3 \pm 5.1$ & $36.8 \pm 9.4$ & $<0.001$ \\
After M $\Delta \mathrm{I}(\mathrm{dB})$ & $32.2 \pm 8.6$ & $17.8 \pm 4.6$ & $<0.001$ \\
\hline Before $\Delta \mathrm{AUC}$ & $227.2 \pm 56.7$ & $1377.2 \pm 205.7$ & $<0.001$ \\
\hline After $\Delta \mathrm{AUC}$ & $2664.1 \pm 456.8$ & $1124.1 \pm 276.4$ & $<0.001$ \\
\hline
\end{tabular}

WT, washout time; RLT, time of relative value of intensity $\geqslant 15$ lasted; Before M $\Delta \mathrm{I}$, max relative value of intensity before washout; After M $\Delta \mathrm{I}$, max relative value of intensity after washout; Before $\triangle \mathrm{AUC}$, relative value of AUC before washout; After $\triangle \mathrm{AUC}$, relative value of AUC after washout.

Currently, there is no report about the CEUS technology of $\mathrm{CHI}$, and this study is the first time to explore the performance of CHI in HCC lesions and its advantages and disadvantages compared with conventional technology of CPS. We reviewed some guidelines for the diagnosis of HCC. European Association for the Study of the Liver (EASL) [7], American Association for the Study of Liver Diseases (AASLD) [8] guidelines, CEUS was not recommended as the standard method of diagnosis and staging for HCC, while the Japanese Society of Hepatology [9] guidelines recognized the diagnostic value of CEUS, but they only accepted Sonazoid (GE, Norway) as the contrast agents due to they had a long post-vascular Kupffer cell specific phase [10].

Sonovue was a purely intravascular agent and its post-vascular phase was negligible and the vascular phase was extremely short, therefore Sonovue was not recommended by the guidelines. We found one of the advantages of CHI was similar to Sonazoid in that it also extended the imaging time, especially the later phase, but their principle is completely different. The former increased the later phase time by expending the range of frequency of nonlinear fundamental and harmonic signal, while the latter depended on the characteristics of the contrast agent of Sonazoid.
Our study has already demonstrated that CHI had a satisfactory enhanced imaging of at least 10 minutes after injection, which not only provided the whole liver scanning time, but also provided the opportunity of relatively late washout detection of some highly differentiated HCC. However, there were some limitations in this study. Firstly, the sample size was relatively small and the study only included a single kind disease of HCC. The differences in CHI performance for different focal liver lesions such as focal nodular hyperplasia (FNH) and cholangiocarcinoma (CCA) needs to be investigated. Besides, most cases of multiple intrahepatic malignancies are metastatic cancers, the superiority of CHI may be more favorable for patients with a metastatic cancer. Therefore, it may have greater significance to explore the value of $\mathrm{CHI}$ in patients with metastatic liver cancer.

In some atypical or rare liver lesions, both CPS and $\mathrm{CHI}$ may misdiagnose them as $\mathrm{HCC}$, as the two misdiagnosed cases in this study. The hemangioma lesion was located under the capsule of the right posterior lobe, and the arterial phase of CPS was affected by respiration and heartbeat and failed to show clear lesion perfusion. Although CHI could show the lesion, it was not described as nodular enhancement in the periphery due to slow perfusion in the arterial phase and the influence 
of respiration. The lesion presented low enhancement with clear margin in the later phase, which led to the misdiagnosis of HCC. The inflammatory lesion was located near the capsule of the right anterior lobe and presented a typical fast-in, fast-out enhancement pattern, however, when we looked back at its two-dimensional image, we found that the lesion was irregularly shaped with blurred margin and obvious parasite-like calcification which was different from HCC.

\section{Conclusion}

The comparison of TIC parameters from the two CEUS methods of CHI and CPS demonstrated their advantages and disadvantages. For HCC, the fast-in perfusion was more obvious by CPS than $\mathrm{CHI}$ before washout, while $\mathrm{CHI}$ provided a more detailed image and longer imaging time after washout. Thus, we recommend the combined use of these two methods for evaluation of hepatic tumors.

\section{Acknowledgment}

This work was supported by grant LQ20H180013 from Natural Science Foundation of Zhejiang Province.

\section{Conflict of Interest}

The authors have no conflict of interest to declare.

\section{References}

[1] El-Serag HB. Hepatocellular carcinoma. N Engl J Med 2011; 365: 1118-27.

[2] Akinyemiju T, Abera S, Ahmed M, Alam N, Alemayohu MA, Allen C, et al. The Burden of Primary Liver Cancer and Underlying Etiologies From 1990 to 2015 at the Global, Regional, and National Level: Results From the Global Burden of Disease Study 2015. JAMA Oncol 2017; 3: 1683-91.

[3] Heimbach JK, Kulik LM, Finn RS, Sirlin CB, Abecassis MM, Roberts LR, et al. AASLD guidelines for the treatment of hepatocellular carcinoma. Hepatology 2018; 67: 358-80.

[4] Zucman-Rossi J, Villanueva A, Nault JC, Llovet JM. Genetic Landscape and Biomarkers of Hepatocellular Carcinoma. Gastroenterology 2015; 149: 1226-39.e4.

[5] Tao SM, Wichmann JL, Schoepf UJ, Fuller SR, Lu GM, Zhang LJ Contrast-induced nephropathy in CT: incidence, risk factors and strategies for prevention. Eur Radiol 2016; 26: 3310-8.

[6] Bartolotta TV, Taibbi A, Midiri M, Lagalla R. Contrast-enhanced ultrasound of hepatocellular carcinoma: where do we stand? Ultrasonography 2019; 38: 200-14.

[7] Josep M Llovet, Michel Ducreux, Riccardo Lencioni, Adrian M Di Bisceglie, Peter R Galle, Jean Francois Dufour, et al. EASLEORTC clinical practice guidelines: management of hepatocellular carcinoma. Eur J Cancer 2012; 48: 599-641.

[8] Bruix J, Sherman M. Management of hepatocellular carcinoma: an update. Hepatology 2011; 53: 1020-2.

[9] Kudo M, Izumi N, Kokudo N, Matsui O, Sakamoto M, Nakashima O, et al. Management of hepatocellular carcinoma in Japan: ConsensusBased Clinical Practice Guidelines proposed by the Japan Society of Hepatology (JSH) 2010 updated version. Dig Dis 2011; 29: 339-64.

[10] Kudo M. Defect Reperfusion Imaging with Sonazoid®: A Breakthrough in Hepatocellular Carcinoma. Liver Cancer 2016; 5: $1-7$. 